\title{
Photoconductivity Decay Kinetics of Acetylferrocene: Effect of Temperature
}

\author{
Raghu Nath Bera \\ ${ }^{1}$ Department of Physics, Basirhat College, Basirhat, \\ North 24 Parganas, West Bengal 743412, India
}

\begin{abstract}
Photoconductivity decay kinetics of a ferrocene $(\mathrm{FcH})$ derivative, acetylferrocene $(\mathrm{AFc})$ were investigated in various temperatures. It was observed that the photocurrent increase to a high value within few seconds after illuminating the sample from a polychromatic light source ( $125 \mathrm{~W}$ mercury lamp). After switching off the light, the current decreases rapidly at the initial stage, then a slow decay of photocurrent was observed with time and finally attains the initial dark current values within few minutes. The kinetics of photoconductivity decay in the case of AFc follows the power law with power less than unity for all measured temperatures. The sublinear dependence of photoconductivity decay indicates exponential type of trap distribution in this ferrocene derivative in the studied temperature range $(289 \mathrm{~K}-303 \mathrm{~K})$. The estimated characteristic temperature $\left(T_{c}\right)$ of exponential trap distribution in the present experiment is not a constant in the studied temperature region but a fluctuation in $T_{c}$ values was noticed with change in temperature. The results have been compared with the previous experiments of dark and photoconductivity of AFc.

Keywords: acetylferrocene, photoconductivity, decay kinetics, trap distribution, recombination
\end{abstract}

\section{Introduction}

The first synthesized organometallic sandwich compound bis $\left(\eta^{5}\right.$-cyclopentadienyl)iron or ferrocene $(\mathrm{FcH})$ attracted much attention due to its potential applications in various technological fields. It has been used efficiently to prepare new material with higher electrical conductivity from poly(vinylidine chloride) [Ozaki J., Watanbe T. and Nishiyoma Y., 1993]. It has also been used to prepare charge transfer compounds from organic solids [Gutmann F., Keyzer H. and Lyons L.E., 1983]. Low frequency electrical current oscillation has been noticed in the case of ferrocene and its derivatives in organic vapour adsorbed state [Mallik B. and Chakraborty A.K., 1996, Bera R. N. and Mallik B., 1997 and
1998]. Photoswitching effect has also been noticed in ferrocene-doped poly(methyl methacrylate) thin films containing chloroform molecules [Basak D. and Mallik B., 2006]. Ferrocene-porphyrin-fullerene triad has been used successfully to construct integrated artificial photosynthetic assemblies for harvesting light and generating photocurrent [Imahori H., Norieda H., Yamada H., Nishimura Y., Yamazaki I., Sakata Y. and Fukuzumi S., 2001]. They are also used in bulk heterojunction solar cell [Patil Y., Misra R., Singhal R. and Sharma G.D., 2017; Patil Y., Misra R., Singh M.K. and Sharma G.D., 2017] and highly efficient dye sensitized solar cells [Daeneke T., Kwon T.-H., Holmes A.B., Duffy N.W. Bach U and Spiccia L., 2011].

\section{Review of Literature}

The studies of photoconductivity of these materials showed some interesting results [Bera R. N. and Mallik B., 1998, Chakraborty A.K., Bera R.N., Bhattacharjee A. and Mallik B., 1998, Chakraborty A.K. and Mallik B., 2009]. Temperature-dependent unusual changes in the factor $(\gamma)$ representing photocurrent-light intensity characteristics for ferrocene and its derivatives have been noticed [Bera R. N. and Mallik B., 1998, Chakraborty A.K., Bera R.N., Bhattacharjee A. and Mallik B., 1998]. Ferrocene-doped poly(methyl methacrylate) thin films containing chloroform molecules showed unusual photoconductivity properties with photoswitching [Basak D. and Mallik B., 2006]. In my earlier communication, I reported the results of photoconductivity decay kinetics of a ferrocene derivative, acetylferrocene (AFc) at a fixed temperature [Bera R. N., 2018]. In this paper I report the effect of temperature on the photoconductivity decay kinetics of acetylferrocene.

\section{Materials and Methods}

Highly pure $\mathrm{AFc}$ was purchased from Aldrich Chem. Co., Inc. (Milwaukee, Wisconsin, USA) in powder 
form and were used after further purification by recrystallization. The experiment was performed using the conventional sandwich cell technique [Rosenberg B., Misra T.N. and Switzer B., 1968]. The pure dry powder of AFc $(10 \mathrm{mg})$ was placed between an indium tin oxide (ITO) coated glass and a stainless-steel electrode and kept inside a brass chamber, fashioned with Teflon. The sample cells were prepared in air but in safe of light. Data were collected by keeping the sample in a controlled dry nitrogen atmosphere. Before any measurements, desorption of water vapour or any pre-adsorbed (if adsorbed during sample cell preparation) vapours/gases from the sample was ensured by repeated heating and cooling treatments of the sample, initially in vacuum and finally in dry nitrogen gas atmosphere. A d.c. bias potential $25 \mathrm{~V}$ across the electrodes was applied from the voltage source of a programmable electrometer (model 617, Keithley Inst. Inc., Cleveland, OH, USA). The current was also recorded by the same electrometer. The temperature of the sample was controlled by a proportional temperature controller (model RTE 110, Neslab Inst. Inc., NH, USA) and was measured by using a copper-constantan thermocouple attached at the top of the metal electrode with the help of a digital millivoltmeter. The details of the experimental set-up can be found elsewhere [Chakraborty A.K., Bera R.N., Bhattacharjee A. and Mallik B.,1998]. A 125 W mercury lamp was used as the polychromatic light source for the photoconductivity measurements. There is a quartz window at the top of the brass chamber for illuminating the sample with light for photoconductivity measurements. The approximate integral photon flux reaching the sample surface was about $3.0 \times 10^{12} \mathrm{~cm}^{-2}$. In this experiment the conductivity cell consisted of two junctions of stainless steel/AFc and AFc/ITO and it was tested that both the junctions were Ohmic in nature.

\section{Results and Discussion}

Before illuminating the sample with light, dark current of the sample was recorded for some time with a fixed bias voltage of $25 \mathrm{~V}$ for different temperatures (289 K- 303 K). From such measurement it was observed that the dark current was more or less constant with time for a fixed bias and fixed temperature. For the photoconductivity decay kinetics study, AFc sample was irradiated with polychromatic light from a $125 \mathrm{~W}$ mercury lamp at different sample temperatures $(289 \mathrm{~K}-303 \mathrm{~K})$. Figure 1 shows the change of current with time before irradiation, during irradiation and after irradiation at a temperature of $289 \mathrm{~K}$. It is noticed that in the initial stage of illumination, the current increases rapidly; then there is a slow rise and finally attains an equilibrium value of current (this is total current $=$ dark current + photocurrent). After switching off the light, the current decreases rapidly at the initial stage and then a slow decay with time and finally attains the initial dark current values within few minutes. This indicates that the photoinduced changes are reversible in nature. The nature of variation of current in AFc sample with irradiation of light for different temperatures is very similar in the studied temperature range (not shown). Photocurrent $\left(I_{p h}\right)$ was estimated by subtracting the dark current $\left(I_{d}\right)$ i.e., current before irradiation from the total current $\left(I_{t}\right)$, i.e., current during irradiation or after switched off light. It is established that the fast decay of photocurrent as observed (within few seconds after switched off the light) in the present experiment arises due to the direct recombination of electron-hole via shallow traps [Pal P., Ghosh D., Jain K.M., Misra T.M., 1987]. The dominant levels having higher capture or release cross-section also contribute to the fast decay of photocurrent. If trapping of carriers freed from traps is negligible then exponential type decay of photocurrent is expected [Bube R.H., 1960]. The slow decay (as observed 20-30 s after switched off light in the present experiment) can be expressed by the power low as given below [Hansmeier, 1974],

$$
\frac{1}{I_{p h}}=k t+c
$$

where, $I_{p h}$ is the photocurrent at time $t ; k$ and $c$ are positive constants. From Eq. (1), we get,

$$
-\log I_{p h}=\log (k t+c)
$$

According to the Eq. (2) a plot of $\log I_{p h}$ vs $\log t$ gives a curved line with instantaneous slope $n>>1$. If $t$ be very large such that $k t>>c$, then Eq. (2) can be written as

$$
I_{p h} \propto t^{-n}
$$

Eq. (3) is known as power low. The value of $n$ can be calculated from the slope of the straight line plot of $\log I_{p h}$ vs $\log t$ following Eq. (3).

In the present experiment, decays of $I_{p h}$ were recorded for different temperatures. Figure 2 shows such decay plots in $\log$-log scale. From Fig.2, it is clear that $I_{p h}$ vs $t$ plot is a straight line in log-log scale. The straight line plot as observed in the present experiment indicates that $k t>>c$ in this case. The values of $n$ were calculated from the slope of the straight line plots of Fig. 2 and are presented in Table-I. The observation of a single straight line in the measured time scale of the present investigation for a fixed temperature indicates that $n$ is constant for the measured time scale for a particular temperature. One interesting observation from this measurement is the variation of $n$ values with temperature. The variation of $n$ values is not systematic with the change of temperature but a fluctuation in $n$ values 
with temperature has been noticed. This observation is consistent with our previous observation where we noticed the fluctuation of $\gamma$ values in the equation $I_{p h}$ proportional to $I_{B}{ }^{\gamma}$ with temperature in the case of $\mathrm{AFc}$ and other ferrocene derivatives [Bera R.N. and Mallik B. 1998]. In the present study of slow decay, the calculated values of $n(0.34$ to 0.49$)$ are lower than unity for all measured temperatures $(289 \mathrm{~K}$ $303 \mathrm{~K})$ indicating the existence of exponential type of trap distribution in the case of AFc. In the previous study [Bera R.N., 2018], I also obtained the value of $n(0.68)$ lower than unity at $284 \mathrm{~K}$. The light intensity dependent photoconductivity study also suggested the presence of exponential type trap distribution in this material [Bera R.N. and Mallik B. 1998]. In contrast, from the measurement of dark conductivity, single discrete level type trap distribution had been reported for this ferrocene derivative [Chakraborty A.K., Bhattacharjee A., and Mallik B., 1994].

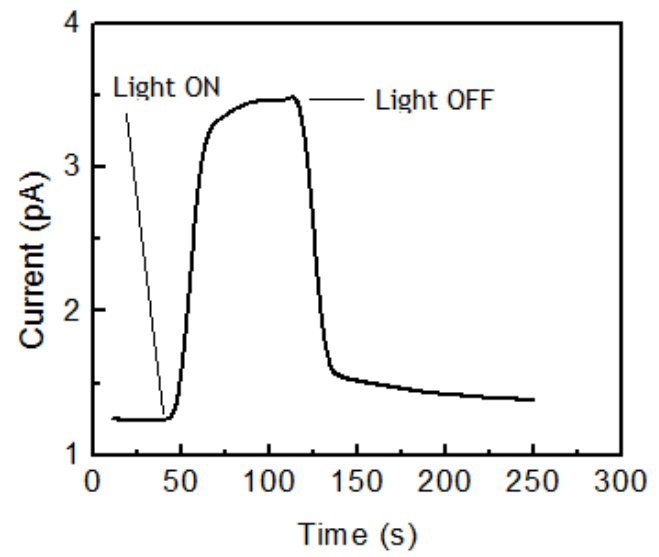

Fig. 1. Photocurrent growth and decay in acetyl ferrocene at a temperature $289 \mathrm{~K}$ with $25 \mathrm{~V}$ bias.

The value of power $n$ in the case of semiconducting material having exponential type trap distribution can be expressed as [Tiedji T. and Rose A., 1981]

$$
n=1-\frac{T}{T_{C}}
$$

Giving rise,

$$
I_{p h} \propto t^{-\left[1-\left(T / T_{C}\right)\right]}
$$

where, $T_{c}$ is a constant known as characteristic temperature for exponential trap distribution. $T_{c}$ gives the rate at which trap density falls with energy. In the present experiment, $T_{c}$ values were calculated following Eq. (4) and are presented in Table-I. The variation of $T_{c}$ values from $452 \mathrm{~K}$ to $594 \mathrm{~K}$ with the variation of temperature has been noticed in the case of $\mathrm{AFc}$ in the present experiment, which is expected as there is a variation of measured $n$ values with temperature.

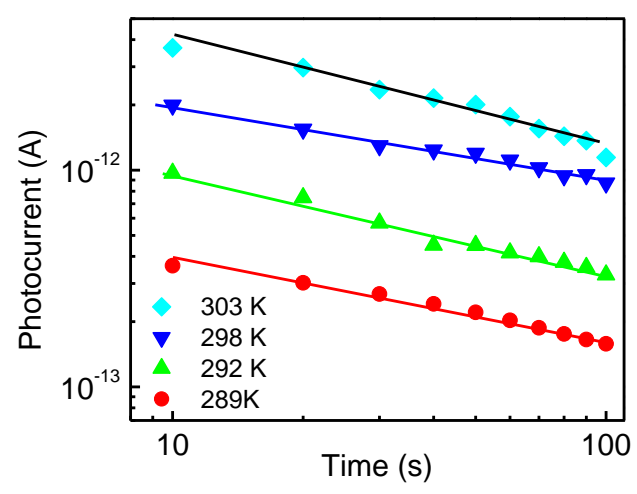

Fig. 2. Dependence of photocurrent on time after switching off the light in acetylferrocene (AFc) for different temperatures.

Table-I The values of characteristic temperature for exponential trap distribution $\left(T_{C}\right)$ and power $(n)$ in the case of acetylferrocene for various measured temperatures

\begin{tabular}{|c|c|c|}
\hline $\begin{array}{c}\text { Temperature } \\
(\mathrm{K})\end{array}$ & $\begin{array}{c}\text { Characteristic } \\
\text { Temperature }(\mathrm{K})\end{array}$ & $\mathrm{n}$ \\
\hline 289 & 458 & 0.37 \\
\hline 292 & 551 & 0.47 \\
\hline 298 & 452 & 0.34 \\
\hline 303 & 594 & 0.49 \\
\hline
\end{tabular}

An $\mathrm{FcH}$ molecule consists of two coplanar cyclopentadienyl rings which are lying above and below an iron atom. This iron atom of an $\mathrm{FcH}$ unit acts as a "ball-bearing" and allows two coplanar cyclopentadienyl rings to rotate with respect to each other about the iron center [Medina J.C., Li C., Bott S.G., Atwood J.L. and Gokel G.W., 1991]. This "ball-bearing" motion allows an $\mathrm{FcH}$ or $\mathrm{AFc}$ molecule to stay in various thermally accessible conformational states at a constant temperature. Physical parameters like heat and illumination by light may changed the conformations of this molecule significantly. The intermolecular interactions among AFc molecules in solid state have also play a crucial role in the photoconductivity change. It is reported that the orientational motion of a AFc molecule may be hindered in solid state due to the intermolecular interactions with neighbouring molecules [Sato K., Katada M., Sano H. and Kono M., 1984]. The changes in conformational motion in the presence of light along with temperature is possibly responsible in the change of photoconductivity behavior in various temperatures in the case of $\mathrm{AFc}$ and hence in the values of $n$ and $T_{c}$.

\section{Conclusions}

In order to investigate the effect of temperature on the photoconductivity decay kinetics of a ferrocene derivative, acetylferrocene (AFc), I studied photoconductivity decay of acetylferrocene in various temperatures. It was observed that the 
photocurrent increases to a high value within few seconds after illumination of the sample with the light from a $125 \mathrm{~W}$ mercury lamp. After turning off the light, the current decreases rapidly at the initial stage, then a slow decay of photocurrent was observed with time and finally attains the initial dark current values within few minutes. A power law dependence of photoconductivity decay has been observed in the case of this ferrocene derivative with a power less than unity. The sublinear dependence of photocurrent decay with time as observed in the present investigation indicates the presence of exponential type trap distribution in this ferrocene derivative. The measured characteristic temperature $\left(T_{c}\right)$ of exponential trap distribution in the present experiment is not a constant in the studied temperature range but a fluctuation in $T_{c}$ values was noticed with change in temperature. The kinetics of photocurrent decay provides us with ideas of trapping/detrapping and recombination processes.

\section{Acknowledgement}

The author is grateful to Professor Avijit Das, Former Head, Department of Spectroscopy, IACS, Kolkata for providing him with the laboratory facilities.

\section{References}

[1] Basak D. and Mallik B., Detailed studies on the photoswitching property of ferrocene-doped poly (methyl methacrylate) thin films containing chloroform molecules, Synthetic Metals vol.156, pp.176-184 (2006)

[2] Bera R. N., Photocurrent decay kinetics of acetylferrocene, International Journal of Advanced Scientific Research and Management, vol.3(8), pp.111-114 (2018).

[3] Bera R. N. and Mallik B., Effects of some physical parameters on the electrical current oscillations in vapor-adsorbed ferrocene, Synthetic Metals, vol. 87, pp.187-192 (1997).

[4] Bera R. N. and Mallik B., Electrical current oscillations in vapor-adsorbed ferrocene derivatives having two substituted groups, Solid State Communications, vol. 108(11), pp. 879-883, (1998).

[5] Bera R. N. and Mallik B., Temperature-dependent unusual changes in the factor $(\gamma)$ representing photocurrent-light intensity characteristics for some ferrocene derivatives, Solid State Communications, Vol. 108(9), pp. 695-700 (1998).

[6] Bube R. H., Photoconductivity of solids (John Wiely and Sons, Inc., New York, 1960 P.275.

[7] Chakraborty A.K., Bera R.N., Bhattacharjee A. and Mallik B., Dark and photoconductive properties of hydroxymethylferrocene, Synthetic Metals, vol.97, pp.63-68 (1998).

\section{ISSN 2455-6378}

[8] Chakraborty A.K., Bhattacharjee A., and Mallik B., Organometalic photoconductors - Dark and photoconductive studies on ferrocene and some of its derivatives, Bulletin of the Chemical Society of Japan, vol.67(3), pp.607-611 (1994).

[9] Chakraborty A.K. and Mallik B., Kinetics of unusual photoinduced currents in the solid state of some ferrocene derivatives, Indian Journal of Physics, vol. 83 (3), pp.291-300 (2009).

[10] Daeneke T., Kwon T.-H., Holmes A.B., Duffy N.W. Bach $U$ and Spiccia L., High-efficiency dyesensitized solar cell with ferrocene-based electrolytes, Nature Chemistry, vol. 3, pp.211-215 (2011).

[11] Gutmann F., Keyzer H. and Lyons L.E., Organic Semiconductors, Robert E. Krieger, Malabar, Florida, p.360, 463 (1983).

[12] Hansmeier, Organic Semiconductors: Dark and Photoconductivity of Organic Solids (Verlag Chemie 2) p.311 (1974).

[13] Imahori H., Norieda H., Yamada H., Nishimura Y., Yamazaki I., Sakata Y. and Fukuzumi S., Lightharvesting and photocurrent generation by gold electrodes modified with mixed self-assembled monolayers of boron-dipyrrin and ferroceneporphyrin-fullerene triad, Journal of the American Chemical Society, vol. 123(1), pp. 100-110 (2001).

[14] Mallik B. and Chakraborty A.K., Low-frequency electrical current oscillations in vapor-adsorbed ferrocene, Journal of physical Chemistry, vol. 100(6), pp.2145-2147 (1996).

[15] Medina J.C., Li C., Bott S.G., Atwood J.L. and Gokel G.W., A molecular receptor based on the ferrocene system: Selective complexation using atomic ballbearings Journal of the American Chemical Society, vol.113(1), pp.366-367 (1991).

[16] Ozaki J., Watanbe T. and Nishiyoma Y., Effect of ferrocene on the carbonization of poly(vinylidene chloride), Journal of Physical Chemistry, vol. 97(7), pp.1400-1405 (1993).

[17] Pal P., Ghosh D., Jain K.M., Misra T.M., Transport of charge carriers in methylbixin crystals: Photoconductivity decay kinetics, Japanese Journal of Applied Physics., vol.26(2), pp.298-299 (1987).

[18] Patil Y., Misra R., Singh M.K. and Sharma G.D., Ferrocene-diketopyrrolopyrrole based small molecule donors for bulk heterojunction solar cells, Physical Chemistry Chemical Physics, vol. 19, pp.7262-7269 (2017).

[19] Patil Y., Misra R., Singhal R. and Sharma G.D., Ferrocene-diketopyrrolopyrrole based nonfullerene acceptors for bulk heterojunction polymer solar cells, Journal of Material Chemistry A, vol. 5, pp.1362513633 (2017).

[20] Rosenberg B., Misra T.N. and Switzer B., Mechanism of olfactory transduction, Nature, vol. 217, pp.423427 (1968).

[21] Sato K., Katada M., Sano H. and Kono M., Studies on the phase transition near the melting point in acetylferrocene, Bulletin of the Chemical Society of Japan, vol.57(9), pp.2361- 2365 (1984). 